\title{
A Comprehensive Cardiopulmonary Simulation Model for the Analysis of Hypercapnic Respiratory Failure
}

\author{
Nicolas W. Chbat, Member, IEEE, Massimo Giannessi, Antonio Albanese, and Mauro Ursino
}

\begin{abstract}
We developed a new comprehensive cardiopulmonary model that takes into account the mutual interactions between the cardiovascular and the respiratory systems along with their short-term regulatory mechanisms. The model includes the heart, systemic and pulmonary circulations, lung mechanics, gas exchange and transport equations, and cardio-ventilatory control. Results show good agreement with published patient data in case of normoxic and hyperoxic hypercapnia simulations. In particular, simulations predict a moderate increase in mean systemic arterial pressure and heart rate, with almost no change in cardiac output, paralleled by a relevant increase in minute ventilation, tidal volume and respiratory rate. The model can represent a valid tool for clinical practice and medical research, providing an alternative way to experience-based clinical decisions.
\end{abstract}

\section{INTRODUCTION}

Hypercapnic respiratory failure is very commonly seen in intensive care units (ICUs). This type of failure could be encountered in patients suffering from chronic obstructive pulmonary disease (COPD), sleep apnea, obesity hypoventilation syndrome, and opiate intoxication. Hypercapnia results from decreased respiratory rate and minute ventilation. Normoxic hypercapnia is a condition where only ventilation is affected while gas exchange remains intact. For instance, opiates suppress respiratory drive of the brain stem, hence decreasing the respiratory rate and subsequently increasing arterial blood $\mathrm{CO}_{2}$ partial pressure $\left(\mathrm{PaCO}_{2}\right)$. Hyperoxic hypercapnia is induced by exposure to high fraction of inspired oxygen $\left(\mathrm{FiO}_{2}\right)$ in patients with chronic $\mathrm{CO}_{2}$ retention such as COPD with chronic air trapping and sleep apnea. These patients have chronic mild hypoxia that continues to stimulate their respiratory drive. Their chemoreflex system is used to chronic low oxygen saturation, as could be seen from an arterial blood gas finger test. When such patients are given high $\mathrm{FiO}_{2}$ via a mask, say, their respiratory drive gets inhibited hence becoming hypercapnic. Hypercapnic

Manuscript received April 23, 2009. This work was supported in part by the Department of Electronics, Computer Science and Systems, University of Bologna (Italy) and by Philips Research North America (NY, USA).

N. W. Chbat is with Philips Research North America, Briarcliff Manor, NY 10510, USA (e-mail: chbat@philips.com).

M. Giannessi is with Department of Electronics, Computer Science and Systems, University of Bologna, Cesena (FC), 47023 Italy. Corresponding author phone: +39.0547.338953; fax: +39.0547.339208; e-mail: massimo.giannessi@unibo.it.

A. Albanese is with Department of Electronics, Computer Science and Systems, University of Bologna, Cesena (FC), 47023 Italy (e-mail: antonio.albanese2@gmail.com).

M. Ursino is with Department of Electronics, Computer Science and Systems, University of Bologna, Bologna, 40136 Italy (e-mail: mauro.ursino@unibo.it). respiratory failure could further be either isolated or associated with hypoxic respiratory failure. Simulation of such conditions with a comprehensive cardiopulmonary model offers invaluable clinical insights.

\section{METHODS}

The cardiopulmonary model (Fig. 1) incorporates cardiovascular circulation, lung mechanics (with distinction among larynx, trachea, bronchi and alveoli), metabolic exchange equations at the alveoli and capillaries, and the control of respiratory rate and tidal volume by central and peripheral chemoreceptors. The model sections are first presented, followed by hypercapnic scenarios.

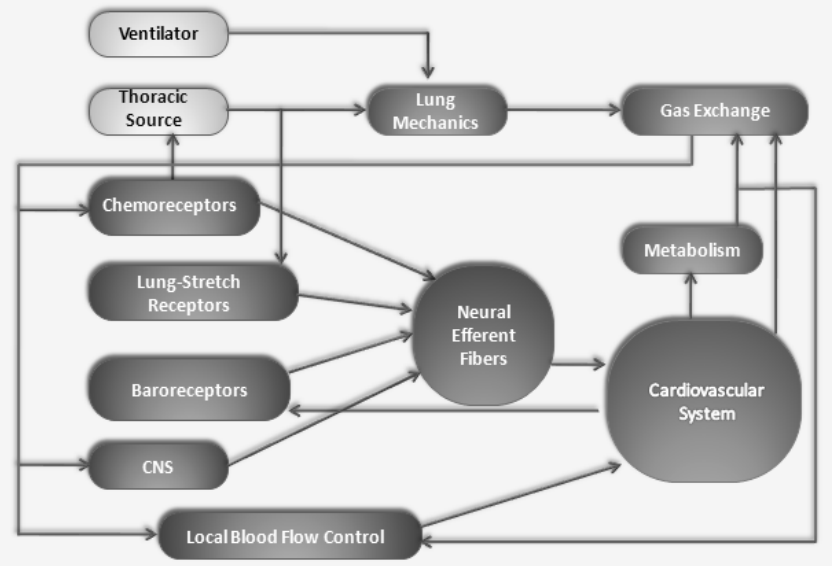

Fig. 1. Cardiopulmonary model diagram.

\section{A. Cardiovascular Model}

The model includes four cardiac chambers with nonlinear ventricular contractility, pulmonary and systemic circulations (see Fig. 2). The latter is subdivided into five districts arranged in parallel (brain, coronary, skeletal muscle, splanchnic and the remaining extrasplanchnic vascular beds).

According to Fig. 2, the different compartments include hemodynamics in the large arteries (systemic and pulmonary), the peripheral circulation, and the venous circulation. Three main kinds of lumped parameters are used in building the model: inertances, which account for inertial forces in blood (these are relevant in large arteries only); resistances, which account for pressure energy losses (this parameter is especially important in the peripheral circulation) and capacitances, which represent the blood volume stored in each compartment (this parameter is especially relevant in the venous circulation). Volume, in turn, is divided in an unstressed volume, which does not 
stretch the vessel wall, and a stressed volume (which causes stress in the wall, and hence is associated with increase in internal pressure). The reader can find details, equations and parameter values in former publications [1], [2].

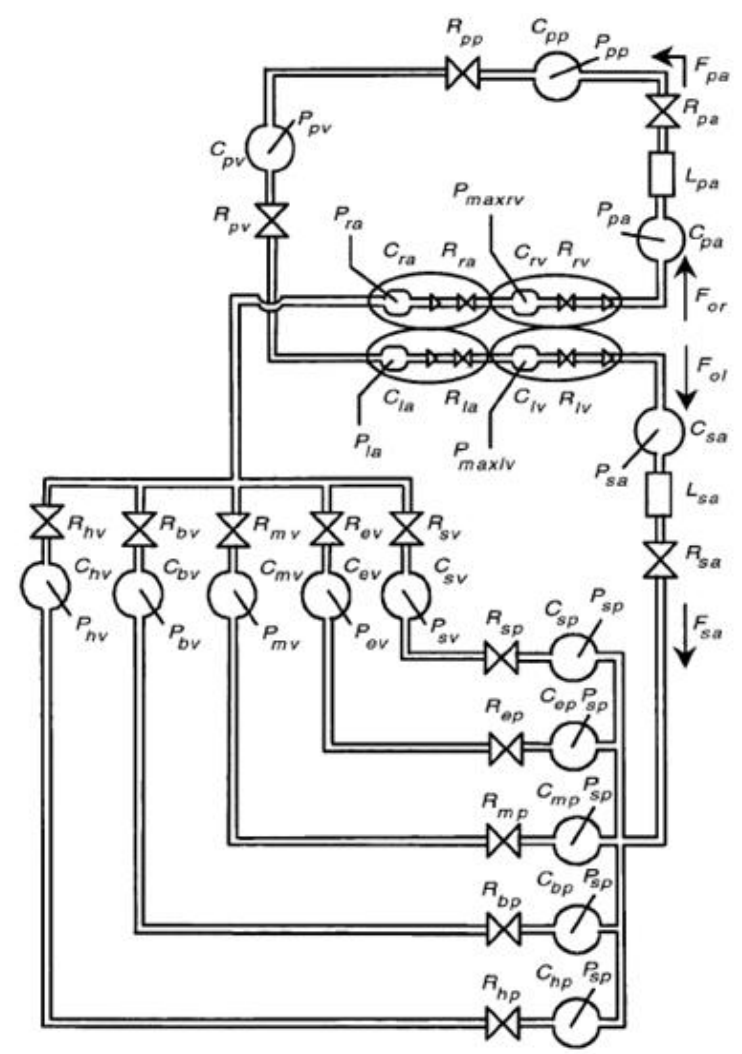

Fig. 2. Hydraulic analog of the model in which resistances are represented with restrictions and compliances with expanded spaces. P, pressures; R, hydraulic resistances; C, compliances; L, inertances; F, flows; sa, systemic arteries; sp and sv, splanchnic peripheral and splanchnic venous circulation; ep and ev, extrasplanchnic peripheral and extrasplanchnic venous circulation; $\mathrm{mp}$ and $\mathrm{mv}$, peripheral and venous circulation in the skeletal muscle vascular bed; bp and bv, peripheral and venous circulation in the brain; $\mathrm{hp}$ and $\mathrm{hv}$, peripheral and venous circulation in the heart (coronary vascular bed); la, left atrium; lv, left ventricle; pa, pulmonary arteries; pp and pv, pulmonary peripheral and pulmonary venous circulation; ra, right atrium; rv, right ventricle.

Short term regulatory mechanisms work via local metabolic factors on peripheral resistances and through the action of the autonomic nervous system. The latter modulates several parameters via two major efferent neural branches, i.e., the sympathoadrenergic and the vagus. Vagal stimulation raises heart period. Sympathetic stimulation causes vasoconstriction in arterioles and veins (thus increasing peripheral resistances and decreasing venous volumes), increases heart contractility and heart rate (HR). Some vascular beds (splanchnic circulation, skeletal muscle circulation and circulation in the other extrasplanchnic districts) are very sensitive to sympathetic stimulation. Conversely, the cerebral and coronary circulations are independent of the autonomic control mechanism. Finally, the sympathetic and vagal neural branches respond to several groups of afferent influences triggered by baroreceptors, chemoreceptors and lung-stretch receptors.

\section{B. Lung mechanics model}

The lung mechanics model has been taken from an existing model in the literature [3]. It combines all parallel branches in both lungs and divides this into four segments in series: larynx, trachea, bronchea and alveoli, together with mouth and nose. As shown in the electrical circuit in Fig. 3, each section has been represented by a linear resistance and a linear capacity, which takes into account the compliance, combining the effects of chest-wall elasticity and the compressibility of air. An unstressed volume has been included for each section. The model is driven by a pressure generator $\left(U_{t}\right)$ assumed to vary sinusoidally, which represents the transmural pressure induced by the respiratory muscles; this pressure is applied to the compliances of the three segments (trachea, bronchea and alveoli) that are within the chest cavity.

According to the suggestion reported in [4], a further forcing function $\left(\mathrm{U}_{\mathrm{m}}\right)$ has been added to the model in [3], in order to reproduce the pressure imposed by an external ventilator to the mouth. In natural breathing condition, the action of the external generator is nullified, whereas it could be restored in order to simulate artificial ventilation condition (in this case also the action of $U_{t}$ could be switch off or superimposed to the ventilator in order to takes into account simultaneous natural and artificial breathing condition).

The model gives as outputs the pressures inside each compartment and their volume, together with the instantaneous air flow into and out of the lung.

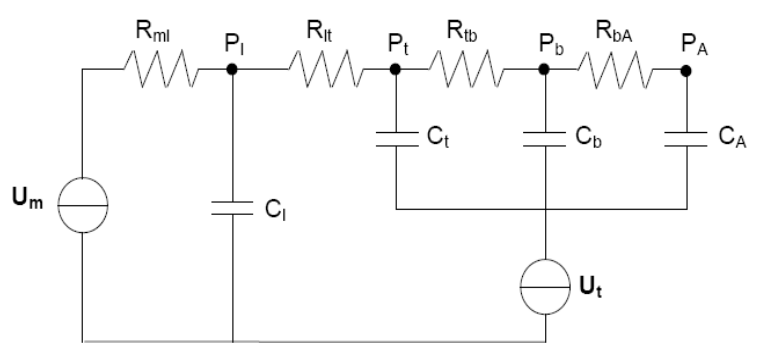

Fig. 3. Electrical analog representing the lung mechanics system. $\mathrm{U}_{\mathrm{m}}$ : external ventilator pressure source; $\mathrm{U}_{\mathrm{t}}$ : respiratory muscles pressure source; $\mathrm{R}_{\mathrm{ml}}$ : resistance of the larynx; $\mathrm{R}_{\mathrm{lt}}$ : resistance of the trachea; $\mathrm{R}_{\mathrm{tb}}$ : resistance of the bronchea; $\mathrm{R}_{\mathrm{bA}}$ : resistance of the alveoli; $\mathrm{C}_{\mathrm{l}}$ : compliance of the larynx; $\mathrm{C}_{\mathrm{t}}$ : compliance of the trachea; $\mathrm{C}_{\mathrm{b}}$ : compliance of the bronchea; $\mathrm{C}_{\mathrm{A}}$ : compliance of the alveoli; $\mathrm{P}_{1}$ : internal pressure of the larynx; $\mathrm{P}_{\mathrm{t}}$ : internal pressure of the trachea; $\mathrm{P}_{\mathrm{b}}$ : internal pressure of the bronchea; $\mathrm{P}_{\mathrm{A}}$ : internal pressure of the alveoli.

\section{Gas exchange model}

The gas exchange model has been derived from an existing model [5] on which several modifications have been performed. In particular, the present model (Fig. 4) splits up the gas exchange processes into three compartments: anatomical dead space, alveoli, and pulmonary capillary. The dead volume is variable because it depends on the changes in volume in the larynx, trachea and bronchi. In addition, the blood flow through the pulmonary capillaries is modelled, and so is the circulation in the pulmonary shunts. 
Inputs to the gas exchange model are:

- air flow into the lung, from the lung mechanics model;

- gas fractions in the inspired air, assumed constant;

- dead space and alveolar volumes, which are outputs of the lung mechanics model;

- blood flows at different levels of the pulmonary vascular bed, which are outputs of the cardiovascular model;

- concentrations of gas in the blood entering the pulmonary peripheral compartment, assumed to be equal to the concentrations of gas in the mixed venous blood that are outputs from the metabolism model. This assumption is justified considering that no generation phenomena happen between the systemic veins and pulmonary peripheral compartment (this is the same assumption used in the metabolism model).

Outputs of the gas exchange model are the gas concentrations in the arterial blood, which are converted to partial gas pressures through the $\mathrm{O}_{2}$ and $\mathrm{CO}_{2}$ dissociations curves, which are also included in the model.

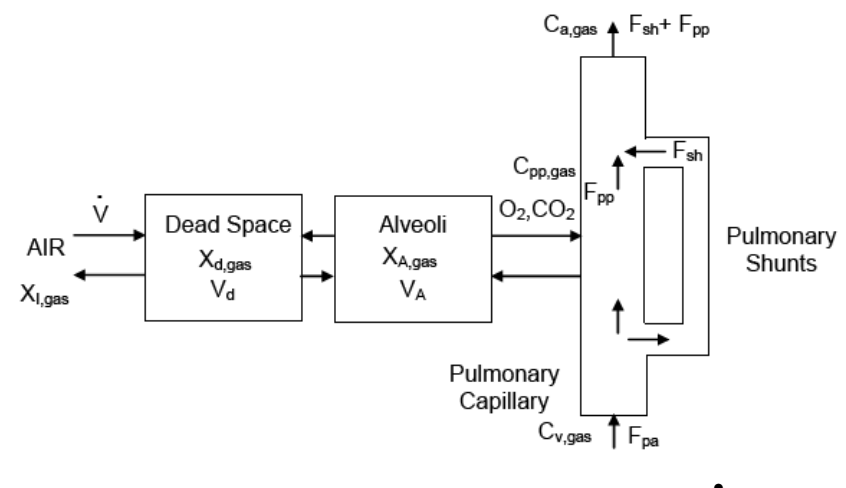

Fig. 4. Block diagram of the integrated gas exchange model. $\mathrm{V}$ : air flow into the lungs; $\mathrm{X}_{\mathrm{I} \text {,gas }}$ : gas fraction in the inspired air; $\mathrm{X}_{\mathrm{d}, \text { gas }}$ : gas fraction in the dead space; $V_{\mathrm{d}}$ : dead space volume; $\mathrm{X}_{\mathrm{d}, \text { gas }}$ : gas fraction in the alveoli; $\mathrm{V}_{\mathrm{A}}$ : alveoli volume; $\mathrm{C}_{\mathrm{v}, \text { gas }}$ : concentrantions of gas in the mixed venous blood; $\mathrm{C}_{\mathrm{pp}, \text { gas }}$ : concentrantions of gas in the pulmonary capillary; $\mathrm{C}_{\mathrm{a}, \mathrm{gas}}$ : concentrantions of gas in the arterial blood; $\mathrm{F}_{\mathrm{pa}}$ : blood flow in the pulmonary arteries; $\mathrm{F}_{\mathrm{pp}}$ : blood flow in the pulmonary capillary; $\mathrm{F}_{\mathrm{sh}}$ : blood flow in the pulmonary shunts.

\section{The respiration control model}

Control of respiration is mediated by the action of both peripheral and central chemoreceptors, since peripheral chemoreceptors are sensitive to both arterial blood $\mathrm{O}_{2}$ partial pressure $\left(\mathrm{PaO}_{2}\right)$ and $\mathrm{PaCO}_{2}$ while central chemoreceptors are sensitive to $\mathrm{PaCO}_{2}$ only.

The model of the respiration control has been significantly modified compared with the one reported in [6], [7]. In that work, chemoreceptors act on respiration by modifying the tidal volume directly, whereas in the present model chemoreceptors act on respiration by modifying the amplitude and the frequency of the thoracic muscles sinusoidal pressure generator $\left(\mathrm{U}_{\mathrm{t}}\right)$.

\section{RESULTS AND DISCUSSION}

In order to illustrate the possibilities offered by the model, in this study, hypercapnic conditions are simulated and compared to published data. The following figures present a comparison between model simulation results and in vivo data, during a condition of normoxic hypercapnia. In particular, Fig. 5 compares the time response of minute ventilation simulated with the model (left panel) and measured by Reynolds et al. [8] (right panel), due to a step increase of $\mathrm{PaCO}_{2}$ from 40 to $56 \mathrm{mmHg}$, lasting 25 minutes. The agreement is acceptable in terms of the amplitude of the steady-state response as well as the time constant $(11 \%$ and $6 \%$ errors respectively).
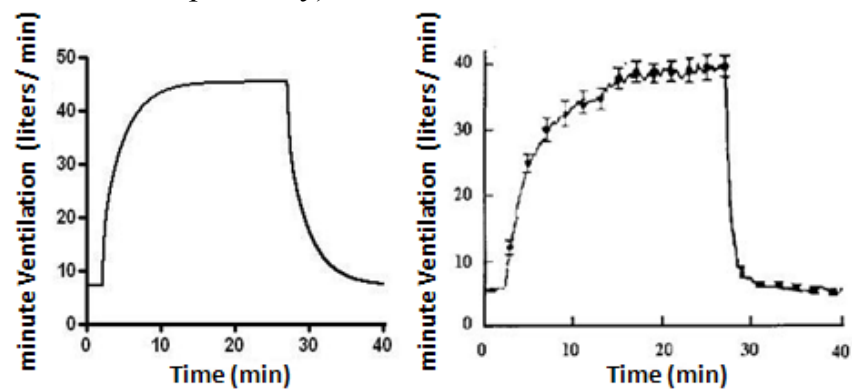

Fig. 5. Time pattern of minute ventilation simulated with the model (left panel) and measured by Reynolds et al. [8] (right panel) in response to a 25 min step normoxic hypercapnia ( $\mathrm{PaCO} 2$ from $40 \mathrm{mmHg}$ to $56 \mathrm{mmHg}$ ). Clinical data are means $\pm \mathrm{SE}$.

Fig. 6 shows the percentage changes in mean systemic arterial blood pressure (MAP), cardiac output (CO), and HR at steady state after normoxic hypercapnia $(10 \mathrm{mmHg}$ step increase in $\mathrm{PaCO}_{2}$ ).

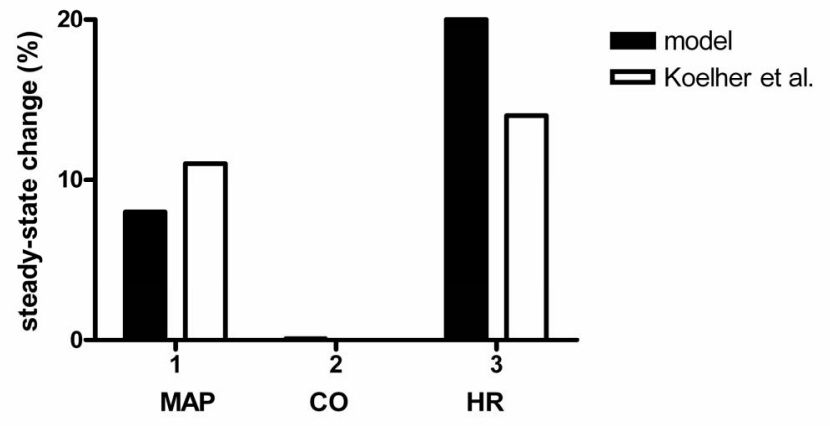

Fig. 6. Steady-state percent changes in MAP, CO, and HR simulated with the model (black bars) and measured by Koehler et al. [9] (white bars) in response to a $10-\mathrm{mmHg} \mathrm{PaCO}_{2}$ increase in normoxic conditions $\left(\mathrm{PaO}_{2}=80\right.$ $\mathrm{mmHg}$ ).

The cardiovascular response also indicated correct trending as compared with data taken from [9].

Fig. 7 compares the responses of the main ventilatory variables, at different levels of hyperoxic hypercapnia, with data reported by Reynols et al. [8]. It shows the correct trending of the respiratory response in terms of minute ventilation, tidal volume, and respiratory rate at different hypercapnic severity levels.

The model also allows for simulation of many other physiological variables at different compartments in the lungs, gas exchange, and metabolism. Another appropriate metabolic variable for hypercapnic respiratory failure is venous concentration of $\mathrm{CO}_{2}$ that is shown in Fig. 8. 

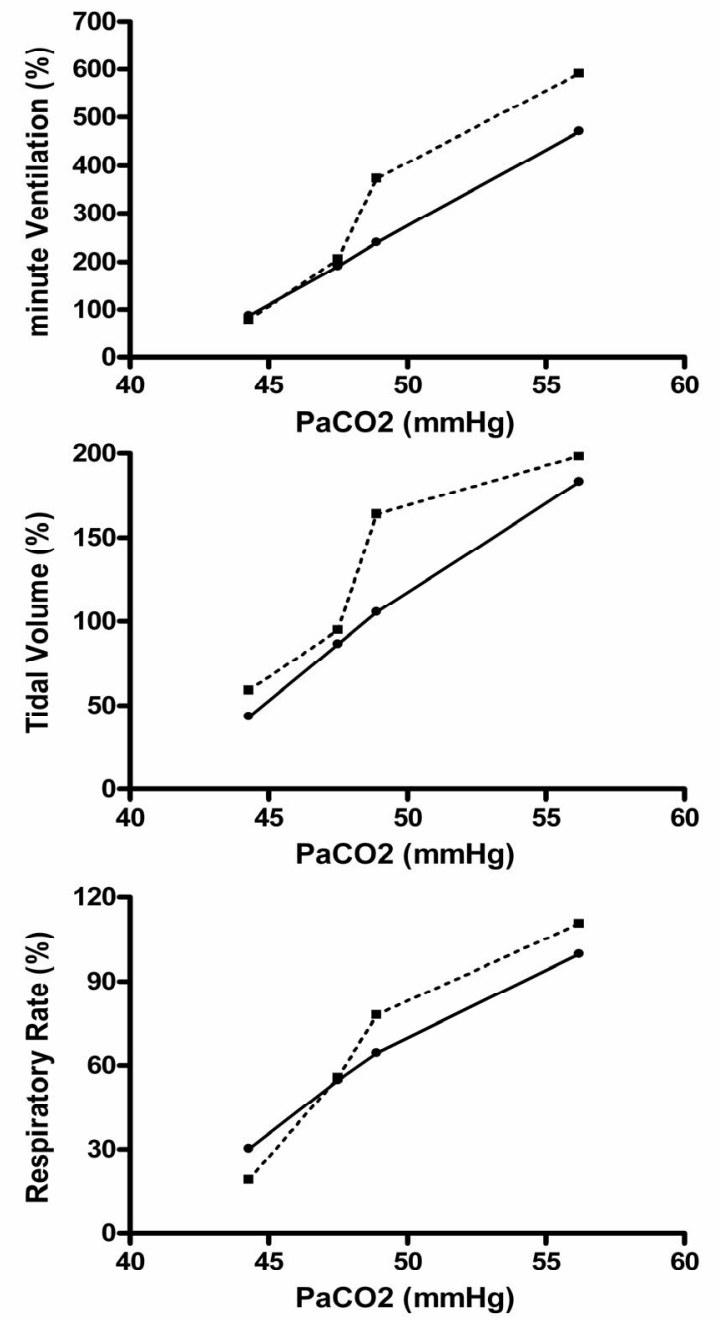

Fig. 7. Steady-state percent changes for minute ventilation, tidal volume and respiratory rate simulated with the model (solid line) and measured by Reynolds et al. [8] (dotted line) in response to different level of hyperoxic hypercapnia.

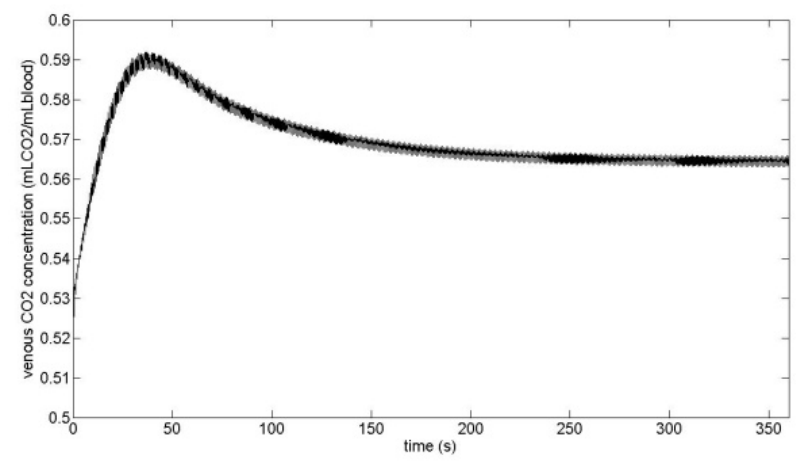

Fig. 8. Mixed venous blood $\mathrm{CO}_{2}$ concentration - normoxic hypercapnia

The response shown is due to a $10 \mathrm{mmHg}$ step increase of $\mathrm{PaCO}_{2}$ while maintaining normal $\mathrm{PaO}_{2}(100 \mathrm{mmHg})$. The venous $\mathrm{CO}_{2}$ concentration increases rapidly at first, but then the respiratory drive increases thus bringing the concentration lower. This dynamic behavior is a function of the central chemoreceptors' time constants. The afferent feedback from these receptors cause the efferent control signals (from the brain) to dictate the amplitude and frequency (drive) of the thoracic muscle actuators $\left(\mathrm{U}_{\mathrm{t}}\right)$. This closed-loop regulator is trying to maintain a normal venous concentration of around $0.525 \mathrm{~mL} / \mathrm{mL}$. However, under this normoxic hypercapnia condition, the actuators saturate resulting in a high steady-state value of $\mathrm{CO}_{2}$ venous concentration of $0.564 \mathrm{~mL} / \mathrm{mL}$ (a plausible scenario for patients suffering from such condition).

\section{CONCLUSION}

Encouraging first results were obtained from this comprehensive cardiopulmonary model when compared to published data. The comparisons between model predictions and real data are acceptable when normal parameter values are given to the model in order to simulate healthy individuals. The study focused on hypercapnic respiratory failure, in particular normoxic and hyperoxic hypercapnia. The model has feedback regulatory mechanisms for chemoreflex, lung stretch, baroreflex, and autoregulation. These regulate a cardiovascular system linked to lung mechanics, gas exchange and metabolism. Simulating these failures has wide applicability in the ICUs for better understanding of COPD, sleep apnea, obesity hypoventilation syndrome, and opiate intoxication.

Moreover, by altering different parameter values, the model can be used to simulate other pathological conditions of clinical relevance, such as cardiogenic shock, cardiac insufficiency, and alterations in respiratory mechanics.

Hence, the model may be used as a validation tool to examine cardiopulmonary and metabolic changes in the ICU, as well as run different therapeutic scenarios.

\section{REFERENCES}

[1] M. Ursino and E. Magosso, "Acute cardiovascular response to isocapnic hypoxia. I. A mathematical model," Am. J. Physiol Heart Circ. Physiol, vol. 279, no. 1, p. H149-H165, July2000.

[2] E. Magosso and M. Ursino, "A mathematical model of $\mathrm{CO} 2$ effect on cardiovascular regulation," Am. J. Physiol Heart Circ. Physiol, vol. 281, no. 5, p. H2036-H2052, Nov.2001.

[3] V. Rideout, "Respiratory System Modeling," in Mathematical and Computer Modeling of Physiological Systems Englewood Cliffs, New Jersey: Prentice-Hall, 1991, pp. 137-143.

[4] T. G. Christensen and C. Draeby, "Modeling the Lung," in Applied Mathematical Models in Human Physiology. J. T. Ottensen, M. S. Olufsen, and J. K. Larsen, Eds. Philadelphia: SIAM, Society for Industrial and Applied Mathematics, 2004, pp. 201-213.

[5] H. Benallal, C. Denis, F. Prieur, and T. Busso, "Modeling of end-tidal and arterial $\mathrm{PCO} 2$ gradient: comparison with experimental data," Med. Sci. Sports Exerc., vol. 34, no. 4, pp. 622-629, Apr.2002.

[6] F. Lioy, B. D. Hanna, and C. Polosa, "CO2-dependent component of the neurogenic vascular tone in the cat," Pflugers Arch., vol. 374, no. 2, pp. 187-191, May1978.

[7] F. Lioy, M. T. Blinkhorn, and C. Garneau, "Regional hemodynamic effects of changes in $\mathrm{PaCO} 2$ in the vagotomized, sino-aortic deafferented rat," J Auton. Nerv. Syst., vol. 12, no. 4, pp. 301-313, Apr. 1985

[8] W. J. Reynolds, H. T. Milhorn, Jr., and G. H. Holloman, Jr., "Transient ventilatory response to graded hypercapnia in man," $J$ Appl. Physiol, vol. 33, no. 1, pp. 47-54, July1972.

[9] R. C. Koehler, B. W. McDonald, and J. A. Krasney, "Influence of $\mathrm{CO} 2$ on cardiovascular response to hypoxia in conscious dogs," Am. $J$ Physiol, vol. 239, no. 4, p. H545-H558, Oct.1980. 